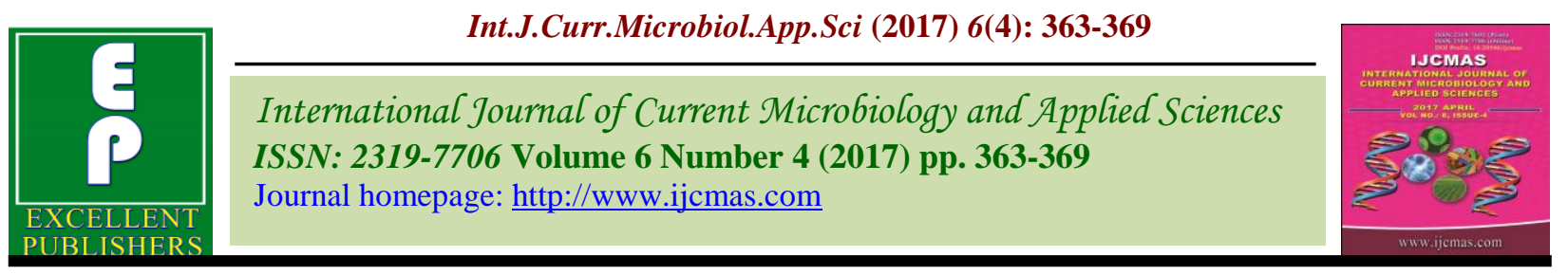

Original Research Article

https://doi.org/10.20546/ijcmas.2017.604.040

\title{
Extension Management Approaches Prevailed in the Voluntary Organization - An Analysis
}

\author{
D.V. Singh* \\ Senior Scientist, Krishi Vigyan Kendra, Kandhamal, OUAT, \\ Bhubaneswar, Odisha, India \\ *Corresponding author
}

\section{A B S T R A C T}

\begin{tabular}{|l|}
\hline Ke y w o r d s \\
Approaches, \\
$\begin{array}{l}\text { Extension } \\
\text { management, Rural } \\
\text { area, Voluntary } \\
\text { organization. }\end{array}$ \\
\hline Article Info \\
\hline $\begin{array}{l}\text { Accepted: } \\
\text { 02 March } 2017 \\
\text { Available Online: } \\
\text { 10 April } 2017\end{array}$ \\
\hline
\end{tabular}

Voluntary organizations are better suited to devote their efforts to foster steady growth in the rural areas. Study has been undertaken in 17 voluntary organizations each in Nabarangpur and Kandhamal districts of Odisha during the year 2014 to access the extent of extension management approaches particularly on planning, empowerment, information system, coordination, supervision, accountability, programme implementation and control process. The findings revealed that 23.25 to $43 \%$ gaps were observed on different aspects of functioning. Better management approaches were prevailed on planning and coordination. Similarly, weaker approaches prevailed on accountability and information system. Socio-personal characteristics of the staffs had not much influence in increasing their capabilities. The voluntary organizations have to analyze their management approaches prevailed, assess weaknesses and take appropriate measures to strengthen the extension management system for successful implementation of programmes.

\section{Introduction}

Management is the process of designing and maintaining an environment in which individuals work together in groups to accomplish the desired goals efficiently (Bhatt, 1995). The voluntary organizations have been established with the basic objectives of serving the rural community and more particularly in remote areas. It has also been time tested that the voluntary organizations are better suited to devote their efforts to foster steady growth in the rural areas (Yousuf et al., 2010). Government machineries have also supported and involving voluntary organizations to assist in the developmental programmes for the upliftment of the rural poor. Unless the voluntary organizations have extension managerial efficiencies suggested programmes could not be implemented successfully for which the present studies have been designed to "Assess the extent of extension management approaches prevailed in the voluntary organizations".

\section{Materials and Methods}

The study was undertaken with 17 voluntary organizations each from Nabarangpur and Kandhamal district of Odisha. One personnel each from administrative, supervisory and field staffs were randomly selected as the respondents from each voluntary organizations making total sample size of 102. Approaches prevailed on planning, 
empowerment, information system, coordination, supervision, accountability, programme implementation and control process were selected as the variables for the study. Information collected on four point scale as very often, often, occasionally and never was analyzed with statistical measures.

\section{Results and Discussion}

Planning lies at the basic of all other managerial functions. Effective planning facilitates timely execution of tasks, provides cooperative and coordinated efforts and makes programme implementation easier. It is observed (Table 1) that the respondents of both the districts were at similar opinion as per the Critical Ratio Value. Management approaches prevailed in the voluntary organizations of Kandhamal district was better than Nabarangpur district. Pooled mean score value revealed that the voluntary organizations have to further strengthened the management approaches since more than $20 \%$ gaps were observed on different aspects of planning.

Empowerment of the staffs is the pre-requisite for successful implementation of activities. The findings revealed that (Table 2) no significant difference was observed in the management approaches on planning among the voluntary organizations of both the district.

However, considerable gap of 20-30\% indicated that the voluntary organization have to further strengthened the capabilities of the staffs particularly on exposing staffs to latest developments, believe their ability in success, expand their vision, provide guidance as well as direction and empowered them to manage the assigned responsibilities.

Voluntary organizations usually depend on the public sector organizations for technological backstopping. Therefore, management of information system is of paramount importance in successful implementation of the activities undertaken. The study revealed (Table 3 ) that significant gaps were observed in the voluntary organizations of both the district on management of different aspects of information system which conclude that the voluntary organizations are giving weightage towards implementation of the activities to achieve the targets for which the impact might not be significant.

Any staff in the organization has to deliver satisfactorily to the job assigned. They have to well acquainted with the environment, develop sincerity, dedication as well as devotion mind and assume responsibility. Therefore accountability is one of the important factors for successful implementation of programmes. But; significant gap of about $40 \%$ were observed (Table 4) on the management approaches towards accountability by the staffs. But the staffs of the voluntary organizations in both the districts were not opined favorably for the acceptance of failures by the head of the organization. Poor responses were also observed on inadequate facilities, insufficient attention to weaker staffs and no appreciation for good work. Unless this approach not exists, staffs cannot assume responsibility and sincerity will not be ascertained.

Voluntary organizations are functioning usually on external funding. The staffs employed do not have much technical competency. Therefore, they have to establish strong coordination with related developmental organizations. But the findings reveal (Table 5) that no significant differences were observed on the coordination approaches prevailed in the voluntary organizations although voluntary organizations of Kandhamal district had better coordination approaches than Nabarangpur district. 
Table.1 Management approaches prevailed on planning

\begin{tabular}{|c|c|c|c|c|c|c|c|c|}
\hline \multirow[t]{2}{*}{$\begin{array}{l}\text { Sl. } \\
\text { No }\end{array}$} & \multirow[t]{2}{*}{ Approach } & \multicolumn{2}{|c|}{$\begin{array}{l}\text { Nabarangpur } \\
\text { District }\end{array}$} & \multicolumn{2}{|c|}{$\begin{array}{l}\text { Kandhamal } \\
\text { District }\end{array}$} & \multirow{2}{*}{$\begin{array}{l}\text { Pooled } \\
\text { Mean } \\
\text { score }\end{array}$} & \multirow[t]{2}{*}{$\begin{array}{l}\text { Gap } \\
(\%)\end{array}$} & \multirow[t]{2}{*}{$\begin{array}{l}\text { C.R. } \\
\text { Value }\end{array}$} \\
\hline & & $\begin{array}{l}\text { Mean } \\
\text { score }\end{array}$ & $\begin{array}{l}\text { Gap } \\
(\%)\end{array}$ & $\begin{array}{l}\text { Mean } \\
\text { score }\end{array}$ & $\begin{array}{l}\text { Gap } \\
(\%)\end{array}$ & & & \\
\hline 1 & Setting long and short term goal & 2.65 & 33.75 & 3.00 & 25.00 & 2.83 & 29.25 & 2.11 \\
\hline 2 & Participatory action plan & 3.00 & 25.00 & 3.22 & 19.50 & 3.11 & 22.25 & 1.27 \\
\hline 3 & $\begin{array}{l}\text { Clearly explaining role of each } \\
\text { staff }\end{array}$ & 3.04 & 24.00 & 3.37 & 15.75 & 3.21 & 19.75 & 1.87 \\
\hline 4 & Exploring resources & 3.02 & 24.50 & 3.24 & 19.00 & 3.13 & 21.75 & 1.26 \\
\hline 5 & $\begin{array}{l}\text { Makes thorough understanding } \\
\text { on programme }\end{array}$ & 3.00 & 25.00 & 3.00 & 25.00 & 3.0 & 25.00 & 0.00 \\
\hline 6 & Provide alternate solutions & 3.12 & 22.00 & 3.14 & 21.50 & 3.13 & 21.75 & 0.11 \\
\hline
\end{tabular}

Table.2 Approaches prevailed on empowerment of staffs

\begin{tabular}{|l|l|l|l|l|l|l|l|l|}
\hline \multirow{2}{*}{$\begin{array}{l}\text { Sl. } \\
\text { No }\end{array}$} & Approach & \multicolumn{2}{l|}{$\begin{array}{l}\text { Nabarangpur } \\
\text { District }\end{array}$} & \multicolumn{2}{l|}{$\begin{array}{l}\text { Kandhamal } \\
\text { District }\end{array}$} & $\begin{array}{l}\text { Pooled } \\
\text { Mean } \\
\text { score }\end{array}$ & $\begin{array}{l}\text { Gap } \\
(\%)\end{array}$ & $\begin{array}{l}\text { C.R. } \\
\text { Value }\end{array}$ \\
\cline { 3 - 10 } & & $\begin{array}{l}\text { Mean } \\
\text { score }\end{array}$ & $\begin{array}{l}\text { Gap } \\
(\%)\end{array}$ & $\begin{array}{l}\text { Mean } \\
\text { score }\end{array}$ & $\begin{array}{l}\text { Gap } \\
(\%)\end{array}$ & & \\
\hline 1 & Expose to latest developments & 2.59 & 35.25 & 2.80 & 30.00 & 2.70 & 32.50 & 1.30 \\
\hline 2 & Expand their vision & 3.06 & 23.50 & 2.61 & 34.75 & 2.84 & 29.00 & 2.71 \\
\hline 3 & Climate of team work & 3.04 & 24.00 & 3.25 & 18.75 & 3.15 & 21.25 & 0.92 \\
\hline 4 & Provides guidance and direction & 2.67 & 33.25 & 3.00 & 25.00 & 2.84 & 29.00 & 1.99 \\
\hline 5 & Sharing information & 3.02 & 24.50 & 3.27 & 18.25 & 3.15 & 21.25 & 1.43 \\
\hline 6 & Believe their ability in success & 2.59 & 35.25 & 3.00 & 25.00 & 2.80 & 30.00 & 2.49 \\
\hline
\end{tabular}

Table.3 Approaches prevailed on management of information system

\begin{tabular}{|c|c|c|c|c|c|c|c|c|}
\hline \multirow[t]{2}{*}{$\begin{array}{l}\text { Sl. } \\
\text { No }\end{array}$} & \multirow[t]{2}{*}{ Approach } & \multicolumn{2}{|c|}{$\begin{array}{l}\text { Nabarangpur } \\
\text { District }\end{array}$} & \multicolumn{2}{|c|}{$\begin{array}{l}\text { Kandhamal } \\
\text { District }\end{array}$} & \multirow{2}{*}{$\begin{array}{l}\text { Pooled } \\
\text { Mean } \\
\text { score }\end{array}$} & \multirow[t]{2}{*}{$\begin{array}{l}\text { Gap } \\
(\%)\end{array}$} & \multirow[t]{2}{*}{$\begin{array}{l}\text { C.R. } \\
\text { Value }\end{array}$} \\
\hline & & $\begin{array}{l}\text { Mean } \\
\text { score }\end{array}$ & $\begin{array}{l}\text { Gap } \\
(\%)\end{array}$ & $\begin{array}{l}\text { Mean } \\
\text { score }\end{array}$ & $\begin{array}{l}\text { Gap } \\
(\%)\end{array}$ & & & \\
\hline 1 & $\begin{array}{lll}\text { Strong mechanism for } \\
\text { receiving information }\end{array}$ & 2.94 & 26.50 & 3.00 & 25.00 & 2.97 & 25.75 & 0.35 \\
\hline 2 & $\begin{array}{l}\text { Information transmitted to all } \\
\text { staffs }\end{array}$ & 2.61 & 34.75 & 2.53 & 36.75 & 2.57 & 35.75 & 0.51 \\
\hline 3 & $\begin{array}{l}\text { Information well understood } \\
\text { by staffs }\end{array}$ & 2.41 & 39.75 & 3.08 & 23.00 & 2.75 & 31.25 & $4.10^{*}$ \\
\hline 4 & $\begin{array}{l}\text { Mechanism for storing } \\
\text { information for reference }\end{array}$ & 2.33 & 41.75 & 2.80 & 30.00 & 2.57 & 35.75 & 2.97 \\
\hline 5 & $\begin{array}{l}\text { Staff empowered in collection } \\
\text { and use of information }\end{array}$ & 2.20 & 45.00 & 2.65 & 33.75 & 2.43 & 39.25 & 2.92 \\
\hline
\end{tabular}

\footnotetext{
*significant 0.05 level
} 
Table.4 Management approaches prevailed on accountability

\begin{tabular}{|l|l|l|l|l|l|l|l|l|}
\hline Sl. & Approach & \multicolumn{2}{|l|}{$\begin{array}{l}\text { Nabarangpur } \\
\text { District }\end{array}$} & \multicolumn{2}{l|}{$\begin{array}{l}\text { Kandhamal } \\
\text { District }\end{array}$} & $\begin{array}{l}\text { Poole } \\
\text { d } \\
\text { Mean } \\
\text { seare }\end{array}$ & $\begin{array}{l}\text { Gap } \\
(\%)\end{array}$ & $\begin{array}{l}\text { C.R. } \\
\text { Value }\end{array}$ \\
\cline { 3 - 11 } & & $\begin{array}{l}\text { Mean } \\
\text { score }\end{array}$ & $\begin{array}{l}\text { Gap } \\
(\%)\end{array}$ & $\begin{array}{l}\text { Mean } \\
\text { score }\end{array}$ & $\begin{array}{l}\text { Gap } \\
(\%)\end{array}$ \\
\hline 1 & Each staff assume responsibility & 1.80 & 55.00 & 2.90 & 27.50 & 2.35 & 41.25 & $3.08^{*}$ \\
\hline 2 & Appreciation for good work & 2.16 & 46.00 & 2.76 & 31.00 & 2.46 & 38.50 & 2.72 \\
\hline 3 & Adequate facilities to staffs & 2.22 & 44.50 & 2.37 & 40.75 & 2.30 & 42.50 & $3.13^{*}$ \\
\hline 4 & More attention to weaker staffs & 2.22 & 44.50 & 2.61 & 34.75 & 2.42 & 39.50 & 2.81 \\
\hline 5 & Head accepts failures & 1.47 & 62.25 & 2.24 & 44.00 & 1.86 & 53.50 & $8.09^{* *}$ \\
\hline
\end{tabular}

*significant at 0.05 level, $* *$ significant 0.01 level

Table.5 Management approaches prevailed on coordination

\begin{tabular}{|l|l|l|l|l|l|l|l|l|}
\hline \multirow{2}{*}{$\begin{array}{l}\text { Sl. } \\
\text { No }\end{array}$} & Approach & \multicolumn{2}{|l|}{$\begin{array}{l}\text { Nabarangpur } \\
\text { District }\end{array}$} & \multicolumn{2}{l|}{$\begin{array}{l}\text { Kandhamal } \\
\text { District }\end{array}$} & $\begin{array}{l}\text { Pooled } \\
\text { Mean } \\
\text { score }\end{array}$ & $\begin{array}{l}\text { Gap } \\
(\%)\end{array}$ & $\begin{array}{l}\text { C.R. } \\
\text { Value }\end{array}$ \\
\cline { 2 - 10 } & $\begin{array}{l}\text { Mean } \\
\text { score }\end{array}$ & $\begin{array}{l}\text { Gap } \\
(\%)\end{array}$ & $\begin{array}{l}\text { Mean } \\
\text { score }\end{array}$ & $\begin{array}{l}\text { Gap } \\
(\%)\end{array}$ & \\
\hline 1 & $\begin{array}{l}\text { Establish good linkage with } \\
\text { district machinery }\end{array}$ & 2.55 & 36.25 & 2.87 & 29.00 & 2.71 & 32.25 & 1.79 \\
\hline 2 & $\begin{array}{l}\text { Coordinate all activities for } \\
\text { optimum resource utilization }\end{array}$ & 3.00 & 25.00 & 3.22 & 19.50 & 3.11 & 22.25 & 1.27 \\
\hline 3 & $\begin{array}{l}\text { Setting coordination for broad } \\
\text { range of goals }\end{array}$ & 2.76 & 31.25 & 2.75 & 31.25 & 2.75 & 31.25 & 0.06 \\
\hline 4 & $\begin{array}{l}\text { Developing internal coordination } \\
\text { matrix }\end{array}$ & 3.12 & 22.00 & 3.27 & 18.25 & 3.23 & 19.25 & 0.85 \\
\hline 5 & $\begin{array}{l}\text { Establishing system of sharing } \\
\text { information }\end{array}$ & 3.10 & 22.50 & 3.35 & 16.25 & 3.23 & 19.25 & 1.41 \\
\hline 6 & $\begin{array}{l}\text { Developing standards for better } \\
\text { coordination }\end{array}$ & 3.00 & 25.00 & 3.14 & 21.50 & 3.07 & 23.25 & 0.81 \\
\hline
\end{tabular}

Table.6 Management approaches prevailed on supervision

\begin{tabular}{|l|l|l|l|l|l|l|l|l|}
\hline $\begin{array}{l}\text { Sl. } \\
\text { No }\end{array}$ & Approach & \multicolumn{2}{|l|}{$\begin{array}{l}\text { Nabarangpur } \\
\text { District }\end{array}$} & \multicolumn{2}{l}{$\begin{array}{l}\text { Kandhamal } \\
\text { District }\end{array}$} & $\begin{array}{l}\text { Pooled } \\
\text { Mean } \\
\text { score }\end{array}$ & $\begin{array}{l}\text { Gap } \\
(\%)\end{array}$ & $\begin{array}{l}\text { C.R. } \\
\text { Value }\end{array}$ \\
\cline { 3 - 10 } & & $\begin{array}{l}\text { Mean } \\
\text { score }\end{array}$ & $\begin{array}{l}\text { Gap } \\
(\%)\end{array}$ & $\begin{array}{l}\text { Mean } \\
\text { score }\end{array}$ & $\begin{array}{l}\text { Gap } \\
(\%)\end{array}$ & \\
\hline 1 & Preparing supervision schedule & 2.20 & 45.00 & 2.82 & 29.50 & 2.51 & 37.25 & $3.96^{*}$ \\
\hline 2 & Periodic performance appraisal & 2.14 & 46.50 & 3.24 & 19.00 & 2.69 & 32.75 & $6.80^{* *}$ \\
\hline 3 & Attention for quality work & 2.96 & 26.00 & 3.00 & 25.00 & 2.98 & 25.50 & 0.24 \\
\hline 4 & Managing problems and conflict & 3.10 & 22.50 & 3.00 & 25.00 & 3.05 & 23.75 & 0.58 \\
\hline 5 & Encourage staffs for suggestions & 3.02 & 24.50 & 3.16 & 21.00 & 2.89 & 27.75 & 0.81 \\
\hline 6 & Creating working environment & 2.61 & 34.75 & 2.86 & 28.50 & 2.74 & 31.50 & 2.68 \\
\hline
\end{tabular}

*significant at 0.05 level, $\quad * *$ significant at 0.01 level 
Table.7 Management approaches prevailed on programme implementation

\begin{tabular}{|l|l|l|l|l|l|l|l|l|}
\hline $\begin{array}{l}\text { Sl. } \\
\text { No }\end{array}$ & Approach & \multicolumn{2}{l|}{$\begin{array}{l}\text { Nabarangpur } \\
\text { District }\end{array}$} & $\begin{array}{l}\text { Kandhamal } \\
\text { District }\end{array}$ & $\begin{array}{l}\text { Pooled } \\
\text { Mean } \\
\text { score }\end{array}$ & $\begin{array}{l}\text { Gap } \\
(\%)\end{array}$ & $\begin{array}{l}\text { C.R. } \\
\text { Value }\end{array}$ \\
\cline { 3 - 9 } & & $\begin{array}{l}\text { Mean } \\
\text { score }\end{array}$ & $\begin{array}{l}\text { Gap } \\
(\%)\end{array}$ & $\begin{array}{l}\text { Mean } \\
\text { score }\end{array}$ & $\begin{array}{l}\text { Gap } \\
(\%)\end{array}$ & & \\
\hline 1 & Pre-arrangement of inputs / materials & 1.80 & 55.00 & 2.48 & 37.75 & 2.14 & 46.50 & $4.76^{*}$ \\
\hline 2 & $\begin{array}{l}\text { Distributing responsibilities among } \\
\text { staffs }\end{array}$ & 3.04 & 23.50 & 3.00 & 25.00 & 3.02 & 24.50 & 0.23 \\
\hline 3 & Closely monitoring each activities & 3.00 & 25.00 & 3.18 & 20.50 & 3.09 & 22.75 & 1.04 \\
\hline 4 & Providing necessary guidance & 2.98 & 25.50 & 3.27 & 18.25 & 3.13 & 21.75 & 1.67 \\
\hline 5 & Adequate mobility to staffs & 3.06 & 23.50 & 3.06 & 23.50 & 3.06 & 23.50 & 0.00 \\
\hline 6 & Solving inconveniency & 3.14 & 21.50 & 3.00 & 25.00 & 3.07 & 23.25 & 0.81 \\
\hline
\end{tabular}

Table.8 Management approaches prevailed on control process

\begin{tabular}{|c|c|c|c|c|c|c|c|c|}
\hline \multirow[t]{2}{*}{$\begin{array}{l}\text { Sl. } \\
\text { No }\end{array}$} & \multirow[t]{2}{*}{ Approach } & \multicolumn{2}{|c|}{$\begin{array}{l}\text { Nabarangpur } \\
\text { District }\end{array}$} & \multicolumn{2}{|c|}{$\begin{array}{l}\text { Kandhamal } \\
\text { District }\end{array}$} & \multirow{2}{*}{$\begin{array}{l}\text { Pooled } \\
\text { Mean } \\
\text { score }\end{array}$} & \multirow[t]{2}{*}{$\begin{array}{l}\text { Gap } \\
(\%)\end{array}$} & \multirow[t]{2}{*}{$\begin{array}{l}\text { C.R. } \\
\text { Value }\end{array}$} \\
\hline & & $\begin{array}{l}\text { Mean } \\
\text { score }\end{array}$ & $\begin{array}{l}\text { Gap } \\
(\%)\end{array}$ & $\begin{array}{l}\text { Mean } \\
\text { score }\end{array}$ & $\begin{array}{l}\text { Gap } \\
(\%)\end{array}$ & & & \\
\hline 1 & Clarity on long term direction & 3.00 & 25.00 & 3.27 & 18.25 & 3.14 & 21.50 & 1.55 \\
\hline 2 & $\begin{array}{l}\text { Modifying goals and objectives as per } \\
\text { the situation }\end{array}$ & 2.82 & 29.50 & 3.16 & 21.00 & 2.99 & 25.25 & 2.00 \\
\hline 3 & Thinks for least cost standards & 3.00 & 25.00 & 3.00 & 25.00 & 3.00 & 25.00 & 0.00 \\
\hline 4 & $\begin{array}{l}\text { Maintaining vertical and horizontal } \\
\text { linkage }\end{array}$ & 1.00 & 75.00 & 2.53 & 36.75 & 1.77 & 55.75 & 11.60 \\
\hline 5 & Framing yardsticks to detect failures & 3.02 & 24.50 & 2.59 & 35.25 & 2.81 & 29.75 & 2.60 \\
\hline 6 & $\begin{array}{ll}\text { Balancing } & \text { socio-organizational } \\
\text { development } & \end{array}$ & 3.00 & 25.00 & 3.37 & 15.75 & 3.19 & 20.25 & 2.11 \\
\hline
\end{tabular}

Table.9 Comparative analysis on prevalence of management approaches

\begin{tabular}{|l|l|l|l|l|l|l|l|}
\hline \multirow{2}{*}{$\begin{array}{l}\text { Sl. } \\
\text { No }\end{array}$} & Approach & \multicolumn{2}{l|}{$\begin{array}{l}\text { Nabarangpur } \\
\text { District }\end{array}$} & \multicolumn{2}{l|}{$\begin{array}{l}\text { Kandhamal } \\
\text { District }\end{array}$} & $\begin{array}{l}\text { Pooled } \\
\text { Mean } \\
\text { score }\end{array}$ & $\begin{array}{l}\text { Gap } \\
(\%)\end{array}$ \\
\cline { 3 - 8 } & & $\begin{array}{l}\text { Mean } \\
\text { score }\end{array}$ & $\begin{array}{l}\text { Gap } \\
(\%)\end{array}$ & $\begin{array}{l}\text { Mean } \\
\text { score }\end{array}$ & $\begin{array}{l}\text { Gap } \\
(\%)\end{array}$ & \\
\hline 1 & Planning & 2.97 & 25.75 & 3.16 & 21.00 & 3.07 & 23.25 \\
\hline 2 & Staff empowerment & 2.83 & 29.25 & 2.99 & 25.25 & 2.91 & 27.25 \\
\hline 3 & Information system & 2.50 & 37.50 & 2.81 & 29.75 & 2.66 & 33.50 \\
\hline 4 & Accountability & 1.97 & 50.75 & 2.58 & 35.50 & 2.28 & 43.00 \\
\hline 5 & Coordination & 2.92 & 27.00 & 3.10 & 22.50 & 3.01 & 24.75 \\
\hline 6 & Supervision & 2.67 & 33.25 & 3.01 & 24.75 & 2.78 & 30.50 \\
\hline 7 & Programme implementation & 2.84 & 29.00 & 3.00 & 25.00 & 2.92 & 27.00 \\
\hline 8 & Control process & 2.64 & 34.00 & 2.99 & 25.25 & 2.81 & 29.75 \\
\hline
\end{tabular}


Table.10 Influence of socio-personal variables on management approaches

\begin{tabular}{|l|l|l|l|l|l|l|l|l|l|l|}
\hline $\begin{array}{l}\text { Sl. } \\
\text { No }\end{array}$ & Approach & Age & \multicolumn{2}{|l|}{ Education } & \multicolumn{2}{l|}{ Experience } & \multicolumn{2}{l|}{ Training } \\
\cline { 2 - 10 } & 'r' & 't' & 'r' & 't' & ' $\mathrm{r}$ ' & 't' & 'r' & 't' \\
\hline 1 & Planning & -0.167 & 1.694 & 0.075 & 0.752 & $-0.310^{* *}$ & 3.260 & -0.055 & 0.551 \\
\hline 2 & $\begin{array}{l}\text { Staff } \\
\text { empowerment }\end{array}$ & -0.140 & 1.424 & $0.339^{* *}$ & 3.603 & $-0.192^{*}$ & 1.957 & 0.092 & 0.924 \\
\hline 3 & $\begin{array}{l}\text { Information } \\
\text { system }\end{array}$ & 0.046 & 0.460 & $-0.332^{* *}$ & 3.521 & -0.114 & 1.148 & -0.060 & 0.600 \\
\hline 4 & Accountability & 0.128 & 1.290 & $-0.265^{*}$ & 2.749 & $0.323^{* *}$ & 3.414 & 0.162 & 1.641 \\
\hline 5 & Coordination & $-0.515^{* *}$ & 6.009 & 0.027 & 0.270 & $0.395^{* *}$ & 4.298 & $-0.218^{*}$ & 2.234 \\
\hline 6 & supervision & -0.213 & 2.180 & $-0.385^{* *}$ & 4.171 & $-0.198^{*}$ & 2.020 & -0.070 & 0.700 \\
\hline 7 & $\begin{array}{l}\text { Programme } \\
\text { implementation }\end{array}$ & 0.062 & 0.621 & 0.112 & 1.127 & 0.115 & 1.158 & $0.324^{* *}$ & 3.425 \\
\hline 8 & Control process & $0.264^{*}$ & 2.736 & $-0.194^{*}$ & 1.978 & $-0.276^{*}$ & 2.872 & $-0.615^{* *}$ & 7.795 \\
\hline
\end{tabular}

*significant at 0.05 level, ${ }^{* *}$ significant at 0.01 level

At the same time, coordination within the voluntary organizations was comparatively better than outside organizations. However, considerable gap of about $20 \%$ and more suggested for further strengthening of coordination approaches succeeding for successful implementation of all activities.

Supervision is an essential part of any organization which substitutes for leadership, authority and contribute to the attainment of the objectives. Significant differential opinions (Table 6) were observed on preparing supervision schedule and periodic performance appraisal indicated that these management approaches not prevailed in all the voluntary organizations. Although, better management approaches prevailed on managing problems and conflicts as well as encouraging staffs for suggestions, but considerable Gap of $24 \%$ and more in all the approaches suggested for further strengthening ensuring better supervision system.

Effective implementation of programmes is the ultimate goal for which all management approaches have to be extended to the staffs of the voluntary organizations. No significant differences were observed (Table 7) on various management approaches prevailed in the voluntary organizations of Nabarangpur and Kandhamal districts. Comparatively better management approaches were prevailed on providing necessary guidance, closely monitoring each activity followed by solving inconveniences, adequate mobility and distributing responsibilities among staffs. Though pre-management of inputs and materials are the pre-requisite for successful implementation of programme, but poor responses observed indicated the deficiencies. On the whole, considerable gap of more than $20 \%$ in different aspects suggested for further strengthening of the management system for better programme implementation.

Each organization has to establish performance standards along with measurement procedure, assessing deviations and provide corrective measures to create good working environment which otherwise called as the control process. Data in table 8 reveal that maintaining vertical and horizontal linkages and insufficient approach for framing yardsticks to detect failures were lacking in the voluntary organizations. Although satisfactory approaches prevailed on other 
aspects, but considerable gap of more than $20 \%$ revealed for further strengthening in management approaches on control process.

Comparative analysis of the management approaches prevailed in the voluntary organizations indicated that (Table 9) voluntary organizations in Kandhamal district had better management approaches than Nabarangpur district. Better management approaches were prevailed on planning and coordination and weakest approaches on accountability as well as information system in comparison to other approaches. However, considerable deficiencies of 23 to $43 \%$ in different approaches suggested for further strengthening of extension management system by the voluntary organizations.

Acquiring knowledge and developing competency are the function of economic, socio-psychological and communication factors. Correlation co-efficient analysis revealed that (Table 10) age, education, experience and training exposure of the respondents had not much influence on increasing their competency in the extension management system. However, education, experience and training had either positive or negative influence on various extension management approaches.

The study on "Extension management approaches prevailed in the voluntary organizations" revealed that voluntary organizations in Nabarangpur district had better management approaches than Kandhamal district. In general, better management approaches were prevailed on planning and coordination. Similarly weaker approaches were prevailed on accountability and information system Socio-personal variables of the respondents had also not much influence in increasing capabilities in various extension management approaches. Moreover, 20 to $34 \%$ deficiencies were observed in the management approaches. The study therefore, conclude that the voluntary organizations have to analyze their existing management approaches, assess the weaknesses and take appropriate measures to strengthen the extension management system enabling to implement various activities successfully.

\section{References}

Bhatt, A. 1995. Voluntary action in India: Roles, trends and challenges, Economics and Political weekly, 30: 870-873.

Elliot, C. 1987. Some aspects of relations between North and South in the NGO sector, World Develop., 15 (suppl): 5768.

Subramanian, V. 1977. Rural Development: The administrative imperatives, Rural Development, Bombay, India Merchants Chamber, Pp 23-28.

Yousuf, M., Imran, A.M.T., Sawar, M. and Naseeruddin, M. 2010. Nongovernment organization's service quality for development of basic education in Pakistan, Africa $J$. Business Manage., 4(14): 3201-3206.

\section{How to cite this article:}

Singh, D.V. 2017. Extension Management Approaches Prevailed in the Voluntary Organization - An Analysis. Int.J.Curr.Microbiol.App.Sci. 6(4): 363-369. doi: https://doi.org/10.20546/ijcmas.2017.604.040 